\title{
Evidence for a diamondlike electronic band structure of Si multilayers on $\mathrm{Ag}(111)$
}

\author{
S. K. Mahatha, ${ }^{1, *}$ P. Moras, ${ }^{1}$ P. M. Sheverdyaeva,,${ }^{1}$ R. Flammini, ${ }^{2}$ K. Horn, ${ }^{3}$ and C. Carbone ${ }^{1}$ \\ ${ }^{1}$ Istituto di Struttura della Materia, Consiglio Nazionale delle Ricerche, I-34149 Trieste, Italy \\ ${ }^{2}$ Istituto di Struttura della Materia, Consiglio Nazionale delle Ricerche, I-00133 Roma, Italy \\ ${ }^{3}$ Fritz-Haber-Institut der Max-Planck-Gesellschaft, Faradayweg 4-6, D-14195 Berlin, Germany
}

(Received 27 October 2015; published 22 December 2015)

\begin{abstract}
Silicon multilayers on $\operatorname{Ag}(111)$ have been suggested to exhibit the structure of silicene, a material that has been heralded as a novel basis for microelectronic applications. However, our angle-resolved photoemission spectra (ARPES) from silicon multilayers on $\mathrm{Ag}(111)$ and of the silver-induced reconstruction of $\mathrm{Si}(111)$ demonstrate, from the close match in the valence level band structures, that the films exhibit a $s p^{3}$ diamondlike structure. This refutes the interpretation of silicon multilayers on $\operatorname{Ag}(111)$ as silicene, a conclusion that is strengthened by the observation from core level photoemission that significant silver segregation occurs to the surface of these layers.
\end{abstract}

DOI: 10.1103/PhysRevB.92.245127

PACS number(s): 73.20.-r, 79.60.-i, 68.35.-p, 68.65.-k

\section{INTRODUCTION}

Silicene [1], a two-dimensional (2D) honeycomb lattice of silicon, is expected to display Dirac cones such as graphene [2-5] with a comparable group velocity [6], and thus may form the basis for a multitude of applications [7]. While freestanding silicene has not been realized, data from silicon films on different surfaces have been interpreted in terms of the presence of honeycomb Si structures, similar to ideal silicene but with various degrees of lattice distortion. The formation of honeycomb monolayers (MLs) has been reported on $\mathrm{Ag}(111)$ [8-18], $\mathrm{ZrB}_{2}(0001)$ [19,20], and $\operatorname{Ir}(111)$ [21]. Si monolayers on $\mathrm{Ag}(111)$ [10-18] have been studied most extensively in this context. This film [22] forms a mixture of $(4 \times 4)$, $(\sqrt{13} \times \sqrt{13}) R 13.9^{\circ}$ and $(2 \sqrt{3} \times 2 \sqrt{3}) R 30^{\circ}$ reconstructed phases, depending on the growth parameters. Such structures present different atomic buckling, arising from the registry relations between silicene and the substrate lattice.

Considerable interest in silicene on $\operatorname{Ag}(111)$ was initially stirred by ARPES investigations of Si MLs that were thought to provide evidence for a Dirac fermion character of the $\pi$ symmetry bands [13], i.e., a linear dispersion as observed for graphene. However, recent studies disprove the existence of Dirac states in a silicene monolayer on $\mathrm{Ag}(111)$, showing that the bands of $\pi$-symmetry lose their $2 \mathrm{D}$ character due to hybridization with the substrate [23]. The spectral features interpreted in earlier studies as silicene Dirac fermions were later found to arise from Ag bulk bands and from an interface state with predominant $\mathrm{Ag} s p$ character; this interface state has a saddlelike shape, rather than a conical dispersion, ruling out its interpretation as a Dirac state [23,24].

The interest in silicene has been renewed by experimental studies reporting the formation of multilayer silicene on $\mathrm{Ag}(111)$ [25-29]. Si films in excess of one monolayer, from bilayers to multilayers of several nanometers, present a $(\sqrt{3} \times \sqrt{3}) R 30^{\circ}$ reconstructed surface in low-energy electron diffraction (LEED) with respect to an ideally flat silicene lattice [12,14-16,25]. ARPES data from these films display a dispersing band near the Fermi energy $\left(E_{\mathrm{F}}\right)$. This band was interpreted as a Dirac cone, leading to a description of the

*Corresponding author: sanjoymahatha@gmail.com film as multilayer silicene $[25,26]$. According to this view, the film consists of weakly interacting Si atomic planes, similar to graphite. These observations may suggest an analogy between silicene on $\mathrm{Ag}(111)$ and graphene on SiC [30], where the Dirac cones form in the second carbon layer but are suppressed in the carbidic layer in contact with the substrate. However, it should be noted that even in graphene bilayers, multilayers, and graphite, the interplane electronic interaction is not negligible and results in a manifold of bands and gap openings, well observable in ARPES [31].

Theoretical studies have addressed the $(\sqrt{3} \times \sqrt{3}) R 30^{\circ}$ structure observed for Si multilayers grown on $\operatorname{Ag}(111)$. A growth mechanism was predicted based on the stacking of dumbbell patterned silicon layers with a $(\sqrt{3} \times \sqrt{3}) R 30^{\circ}$ periodicity [32]. The predicted structure exhibits characteristic features of layered materials, with stronger in-plane than out-of plane bonding. These findings suggest an interpretation for the reported structural LEED and scanning tunneling microscopy (STM) properties of silicene multilayers. However, the calculated electronic band structure displays a band gap larger than in diamondlike $\mathrm{Si}$, while in ARPES an electronic band crosses the Fermi energy [25].

The ARPES results also appear to conflict with information emerging from structural investigations. A comprehensive study including STM, scanning tunneling spectroscopy, atomic force microscopy, and Raman measurements, identifies the Si films as a Ag terminated diamondlike Si(111) structure, contradicting the description of the films as multilayer silicene [33]. Moreover, recent optical reflectance, STM, and photoemission results also conclude that diamondlike silicon is present in this system and indicate that $\mathrm{Ag}$ acts as surfactant [34-36]. Another STM study and theoretical results claim, on the contrary, that the $(\sqrt{3} \times \sqrt{3}) R 30^{\circ}$ honeycomb structure forms on pure diamondlike $\mathrm{Si}(111)$ thin films [37,38]. The $I-V$ LEED curves of a Si multilayer grown on a silver film are found to be similar to that of Ag terminated $\mathrm{Si}(111)$ [39]. However, since the band dispersion data obtained by ARPES were in conflict with such a structural model, it was suggested that multilayer silicene on $\operatorname{Ag}(111)$ might only be fabricated in a narrow temperature range [25], explaining the conflicting observations from LEED and other structural results with the ARPES data. 
It is very important to resolve this conflict of interpretation, and to investigate the electronic structure of multilayer silicene on $\operatorname{Ag}(111)$ prepared at different temperatures in order to clarify the situation. Here we report ARPES and core level photoemission data for different growth temperatures, and over a wide range of $k$ space, with the aim of directly addressing the contrasting findings on electronic and structural properties. Our ARPES data reproduce the band structure reported in earlier investigations; however, we arrive at an entirely different interpretation. Contrary to earlier studies, we show that the ARPES results do not provide evidence for the presence of Dirac cones, and do not establish the formation of multilayer silicene. Rather, we demonstrate that the band structure of the films closely corresponds to that of a $(\sqrt{3} \times \sqrt{3}) R 30^{\circ}-\mathrm{Ag}$ terminated $\mathrm{Si}(111)$ surface, in line with the conclusions of structural investigations. Core level spectra give corresponding and direct evidence for $\mathrm{Ag}$ segregation to the surface of the Si films which is responsible for the $(\sqrt{3} \times \sqrt{3}) R 30^{\circ}$ phase in the Si films.

\section{EXPERIMENTAL METHODS}

ARPES and core level photoemission spectroscopy measurements were performed at the VUV-Photoemission beamline of the Elettra synchrotron in Trieste. The Scienta R4000 electron spectrometer was placed at an angle of $45^{\circ}$ with respect to the direction of the $p$-polarized photon beam. Photoelectrons were collected within the light scattering plane. All photoemission measurements were performed at room temperature with an angular resolution better than $0.3^{\circ}$ and energy resolution of $30 \mathrm{meV}$. The $\mathrm{Ag}(111)$ surface was prepared by cycles of sputtering and annealing at $550{ }^{\circ} \mathrm{C}$ in order to generate an ordered and clean surface, as confirmed by LEED. Si was deposited by resistive heating of a $\mathrm{Si}$ wafer at a rate of $0.01 \mathrm{ML} / \mathrm{min}$ on clean $\mathrm{Ag}(111)$ surface, in accordance with the rate used in other studies $[9,10,14,15,18]$. Here one monolayer of $\mathrm{Si}$ is determined as the quantity corresponding to the completion of the $\mathrm{Si}$ wetting layer at $\sim 240^{\circ} \mathrm{C}$, according to Ref. [22]. The LEED patterns for monolayer silicene were in agreement with the results of Ref. [22]. We grew several wedges of different thicknesses of Si film on $\operatorname{Ag}(111)$ at $\sim 200^{\circ} \mathrm{C}, \sim 235^{\circ} \mathrm{C}$, and $\sim 270^{\circ} \mathrm{C}$ to achieve different dominant phases of monolayer silicene, upon which Si multilayers were grown. The $(\sqrt{3} \times \sqrt{3}) R 30^{\circ}$ pattern was observed for deposition of $\mathrm{Si}$ exceeding one monolayer of coverage. This $(\sqrt{3} \times \sqrt{3}) R 30^{\circ}$ structure grows in registry with the monolayer lattice, producing a distribution of domains oriented in different directions, depending on the deposition temperature as revealed by the LEED pattern. As shown in Ref. [22], at $\sim 200^{\circ} \mathrm{C}$ the monolayer is predominantly $(\sqrt{13} \times \sqrt{13}) R 13.9^{\circ}$, at $\sim 235^{\circ} \mathrm{C}(4 \times 4)$, and at $\sim 270^{\circ} \mathrm{C}$ $(2 \sqrt{3} \times 2 \sqrt{3}) R 30^{\circ}$. The presence of domains with different azimuthal orientation results in a corresponding $k$ broadening of the ARPES spectral features. The azimuthal spread of the $(\sqrt{3} \times \sqrt{3}) R 30^{\circ}$ domains is larger for the films grown at $\sim 200^{\circ} \mathrm{C}$, with respect to those produced at $\sim 235^{\circ} \mathrm{C}$ and at $\sim 270^{\circ} \mathrm{C}$.

The morphology of the films was addressed in previous STM studies. A Si multilayer does not grow as a film of uniform thickness [26-28,33]. STM line profiles show that the growth results in distribution of flat areas of different film thicknesses. Core level data were acquired with photon energy of $150 \mathrm{eV}$ for $\mathrm{Si} 2 p$ and $430 \mathrm{eV}$ for the $\mathrm{Ag} 3 d$ core level. ARPES spectra of multilayer $\mathrm{Si}$ with different thicknesses were measured using $126 \mathrm{eV}$ photon energy. The $(\sqrt{3} \times \sqrt{3}) R 30^{\circ}-\mathrm{Ag} / \mathrm{Si}(111)$ surface was prepared by deposition of $\sim 1$ monolayer of $\mathrm{Ag}$ onto a clean $\mathrm{Si}(111)$ $(7 \times 7)$ surface at room temperature, followed by annealing to $\sim 600^{\circ} \mathrm{C}$. The binding energy of all spectra was calibrated by determining $E_{\mathrm{F}}$ on clean $\operatorname{Ag}(111)$ surfaces.

\section{RESULTS AND DISCUSSION}

\section{A. ARPES and the valence band structure of multilayer silicon on $\operatorname{Ag}(111)$}

The enormous interest in silicon layers exhibiting a silicene structure obviously stems from the possible existence of massless charge carriers, leading to extremely high carrier mobilities, in analogy to graphene; moreover, the possibility to readily introduce such a material into the well-established silicon device manufacturing technology has fired the imagination. Hence it is most important to closely examine those features that were previously interpreted in terms of linearly dispersing Dirac cones. A particular aspect of our study is the direct comparison of the band structure of Si multilayer films on $\operatorname{Ag}(111)$ by ARPES and the one of $(\sqrt{3} \times \sqrt{3}) R 30^{\circ}$ $\mathrm{Ag} / \mathrm{Si}(111)$ structure, since the latter was identified to prevail in silicon films on $\operatorname{Ag}(111)$, suggesting the segregation of $\mathrm{Ag}$ atoms to the surface of the film.

We show ARPES data along $\bar{\Gamma}_{\text {Ag }}-\bar{K}_{\text {Ag }}$ symmetry direction of Si films $(\sim 6 \mathrm{ML})$, grown at $200^{\circ} \mathrm{C}, 235^{\circ} \mathrm{C}$, and $270^{\circ} \mathrm{C}$ in Figs. 1(a)-1(c). For a direct comparison, the band structure of $(\sqrt{3} \times \sqrt{3}) R 30^{\circ}-\mathrm{Ag}$ terminated $\mathrm{Si}(111)$ measured under the same experimental conditions is displayed in Fig. 1(d). A closer inspection of the bands can be obtained from the first derivative of the data as shown in Figs. 1(i)-1(1), revealing the dispersing features in Figs. 1(a)-1(d). In order to provide a quantitative assessment of the evolution of band position and, more importantly, band intensities for the different preparations, we show in Figs. 1(e)-1(h) momentum distribution curves (MDCs) at 1 and $2 \mathrm{eV}$ binding energy obtained from the data in Figs. 1(a)-1(d). The ARPES data show a similar band structure in all four cases. The bands of the Si films grown at different temperatures exhibit some minor differences among themselves, which reflect a different degree of crystalline ordering and diverse morphology, resulting from kinetic effects in the growth processes. The bands of the film grown at $\sim 200{ }^{\circ} \mathrm{C}$ are more diffuse in comparison to those of the other two films. The band sharpness can be related to the degree of crystalline order and finds correspondence in the LEED observations. Another difference concerns the presence of $s p$-derived Ag bands in the films' spectra. They give rise to highly dispersing bands, crossing $E_{\mathrm{F}}$ at $\pm 1.1 \AA^{-1}$, which are more intense for the film grown at $\sim 270{ }^{\circ} \mathrm{C}$ [Fig. 1(c)]. The observation of these bands is supported by the core level results (see Sec. III B) showing that a significant part of the substrate is uncovered for the higher temperature growth, since the $\mathrm{Ag}$ emission is more attenuated at lower temperatures. From a comparison of the MDC data in Figs. 1(e)-1(h), it appears that 


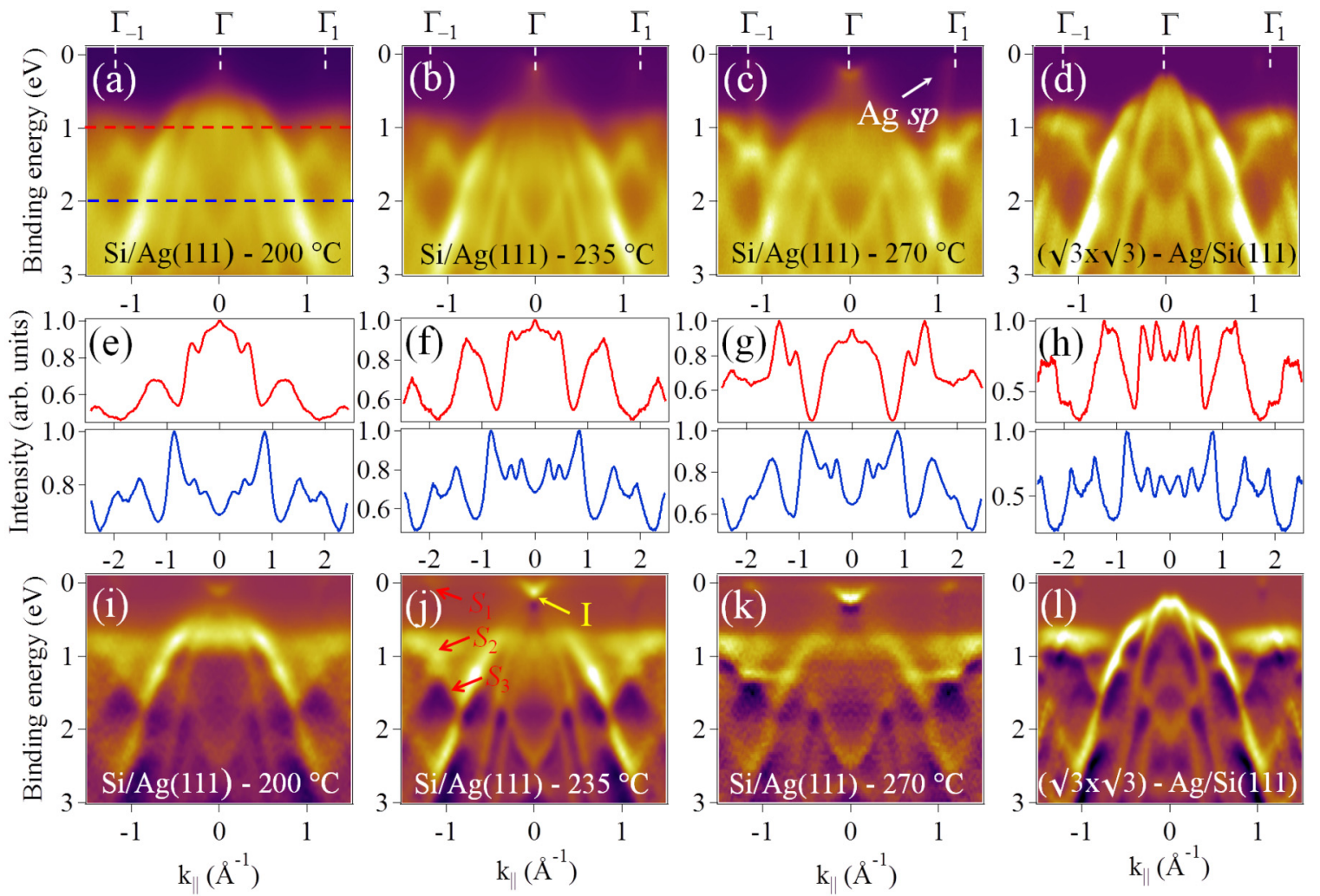

FIG. 1. (Color online) Angle-resolved photoemission maps for multilayer $\mathrm{Si} / \mathrm{Ag}(111)$ along $\bar{\Gamma}_{\mathrm{Ag}}-\bar{K}_{\mathrm{Ag}}$ measured using a photon energy of $126 \mathrm{eV}$ and grown at (a) $200^{\circ} \mathrm{C}$, (b) $235^{\circ} \mathrm{C}$, and (c) $270^{\circ} \mathrm{C}$ and of (d) $(\sqrt{3} \times \sqrt{3}) R 30^{\circ}-\mathrm{Ag}$ on a Si(111) surface along the $\bar{\Gamma}_{\mathrm{Si}}-\bar{K}_{\mathrm{Si}}$ symmetry direction using a photon energy of $126 \mathrm{eV}$. Momentum distribution curves (e)-(h) in extended $k_{\|}= \pm 2.5 \AA^{-1}$ scale of (a)-(d) at $1 \mathrm{eV}$ (upper panel with red line) and $2 \mathrm{eV}$ (lower panel with blue line) binding energy, respectively. First derivative spectra of (a)-(d) has been shown in (i)-(1), respectively, to better resolve the weak features. The white arrow indicates the Ag $s p$ band, the yellow arrow indicates the Ag $L$-gap interface state $(I)$, and the red arrows indicate different $(\sqrt{3} \times \sqrt{3}) R 30^{\circ}$-Ag surface states.

a very good correspondence between the bands from the silicon layers and the $(\sqrt{3} \times \sqrt{3}) R 30^{\circ}-\mathrm{Ag}$ on the $\mathrm{Si}(111)$ surface is already obtained for a preparation temperature of $235^{\circ} \mathrm{C}$. The one-to-one correspondence of peak positions and shapes in $k_{\|}$for a given energy, with minor deviations in relative peak intensities, gives additional evidence of the close resemblance of the band structures.

We note that surface states of the $(\sqrt{3} \times \sqrt{3}) R 30^{\circ}-\mathrm{Ag}$ terminated $\mathrm{Si}(111)$, which are labeled $S_{1}, S_{2}$, and $S_{3}$ according to Refs. [40,41], are observed near the $\bar{\Gamma}_{1,-1}$ (with a wave vector of $\left.\boldsymbol{k}_{\|}= \pm 1.1 \AA^{-1}\right)$ points of the $(\sqrt{3} \times \sqrt{3}) R 30^{\circ}$ surface Brillouin zone. These surface states are also visible in Figs. 1(b) and 1(c) for Si films grown at $\sim 235^{\circ} \mathrm{C}$ and $\sim 270^{\circ} \mathrm{C}$. At $\sim 200^{\circ} \mathrm{C}$ the surface states are still observable, although they are weaker, due to the larger azimuthal broadening, reflecting the domain structure of the Si film. $S_{1}$ is characteristic of $(\sqrt{3} \times \sqrt{3}) R 30^{\circ}-\mathrm{Ag}$ terminated $\mathrm{Si}(111)$, observed close to $E_{\mathrm{F}}$ at $1.1 \AA^{-1}\left(\bar{\Gamma}_{1,-1}\right)$. The position of this state is known to critically depend on a minor deviation from the nominal surface composition. While the state is empty for a precise stoichiometry, a slight excess of Ag content induces a charge doping that shifts the state into the occupied range of the band structure and converts the semiconducting surface into a metal. Features that can be attributed to such a state are found in all films shown here, as well as in the $(\sqrt{3} \times \sqrt{3}) R 30^{\circ}-\mathrm{Ag}$ terminated $\mathrm{Si}(111)$ spectra, as described in more detail in the following, at binding energies varying between 0.2 and $0.4 \mathrm{eV}$, corresponding to an Ag excess in the order of 0.002-0.02 per $\mathrm{Si}(111)$ unit cell [42].

Previous investigations [25,26], which examined the band structure over a more limited energy and momentum range, concluded that the Si films have characteristic features of silicene multilayers, with a weak interplanar interaction. Bands observed at $\bar{\Gamma}$ as well as their replica at corresponding wave vector $\left(\bar{\Gamma}_{1,-1}\right)$ had been interpreted as $\pi$ and $\pi *$ Dirac cones of mono- and multilayer silicene. The strong similarity between the bands of $\mathrm{Si}$ films and the $(\sqrt{3} \times \sqrt{3}) R 30^{\circ}-\mathrm{Ag} / \mathrm{Si}(111)$ leads us to reexamine earlier interpretations of the ARPES results. While our results reproduce the features observed in previous studies [25,26], apart from minor differences related to the much lower Si film thicknesses used in Refs. [25,26], leading to a much reduced contribution from bulk Si valence states, we find that they are readily explained through the comparison with the $(\sqrt{3} \times \sqrt{3}) R 30^{\circ}$-Ag terminated $\mathrm{Si}(111)$ bands and by taking into account the emission from the $\mathrm{Ag}$ substrate. Consider first the region near $\bar{\Gamma}$ which shows three strongly downwards dispersing bands. The two inner curves correspond to those measured in previous studies, within the limited wave vector and energy range there explored. Those two downward-dispersing bands near $\bar{\Gamma}$ were interpreted as $\pi$ Dirac bands of multilayer and monolayer silicene [25,26]. We observe very similar dispersing bands also in the electronic 


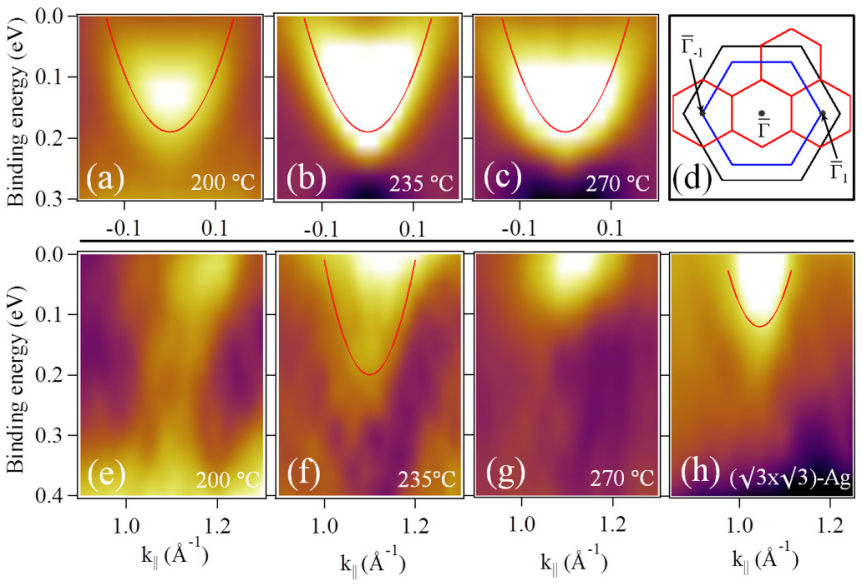

FIG. 2. (Color online) Band dispersion (first derivative spectra along the energy axes to increase the contrast) of multilayer $\mathrm{Si} / \mathrm{Ag}(111)$ grown at $[(\mathrm{b}),(\mathrm{e})] 200^{\circ} \mathrm{C},[(\mathrm{c}),(\mathrm{f})] 235^{\circ} \mathrm{C}$, and $[(\mathrm{d})$, (g) $270^{\circ} \mathrm{C}$, around $\bar{\Gamma}$ and $\bar{\Gamma}_{1}$ and of (h) $(\sqrt{3} \times \sqrt{3}) R 30^{\circ}-\mathrm{Ag}$ on a $\operatorname{Si}(111)$ surface around $\bar{\Gamma}_{1}$ measured using a photon energy of $126 \mathrm{eV}$, along with a parabolic dispersion fitting (solid red line) of clean $\mathrm{Ag}$ surface state [(a)-(c)] and of $S_{1}$ surface state [(f), (h)]. (d) Surface Brillouin zones of $(1 \times 1)-\mathrm{Ag},(1 \times 1)-\mathrm{Si}$, and $(\sqrt{3} \times \sqrt{3}) R 30^{\circ}$-Si are shown as black, blue, and red hexagons, respectively.

structure of $(\sqrt{3} \times \sqrt{3}) R 30^{\circ}-\mathrm{Ag}$ terminated $\mathrm{Si}(111)$. They are known to arise from the heavy-hole, light-hole, and split-off bands, originating from $s p^{3}$ hybridized Si orbitals [43]. Some minor differences with the bulk $\mathrm{Si}$ are likely to arise from finite size and structural distortion of the thin films.

The nearly parabolic band close to $E_{\mathrm{F}}$, which was assigned to the $\pi^{*}$ Dirac band of multilayer silicene [25,26], is also observed in the present data (Fig. 1). Shirai et al. concluded that this band could not be attributed to any of the $(\sqrt{3} \times \sqrt{3}) R 30^{\circ}$ Ag surface states [39], because the effective mass is different from that of the $(\sqrt{3} \times \sqrt{3}) R 30^{\circ}-\mathrm{Ag}$ surface states close to $E_{\mathrm{F}}$. This was interpreted as an indication that, through a fine tuning of the substrate temperature, it could be possible to prepare silicene multilayers. However, we find that that the effective mass $\left(m^{*} / m=0.4\right.$, where $m$ is the mass of free electron) determined from the band dispersion of this state closely corresponds to that of the $\operatorname{Ag}(111)$ Shockley surface state at room temperature; a difference in binding energy at the bottom of the band $(\sim 190 \mathrm{meV})$ compared to that of the surface state on clean $\operatorname{Ag}(111)(63 \mathrm{meV}$ at low temperature) [44] may be related to the influence of $p$-type doping by the adjacent silicon atoms. The binding energy shift suggests that the Shockley surface state could develop into an interface state inside the $310 \mathrm{meV} L$ gap of the $\operatorname{Ag}(111)$ surface, similarly to the case of an interface state observed in a silicene monolayer [23]. The band is weak but visible in the first derivative spectra for all films shown in Figs. 2(a)-2(c). Like other Ag-derived features, its intensity is stronger for samples grown at $\sim 270^{\circ} \mathrm{C}$, as expected, because the silicene leaves larger parts of the $\operatorname{Ag}(111)$ surface partially or fully uncovered. A replica of the surface states at $\bar{\Gamma}$ should appear at $\bar{\Gamma}_{1,-1}$, due to the $(\sqrt{3} \times \sqrt{3}) R 30^{\circ}$ periodicity, common to the Si films and to the $(\sqrt{3} \times \sqrt{3}) R 30^{\circ}$-Ag terminated surface

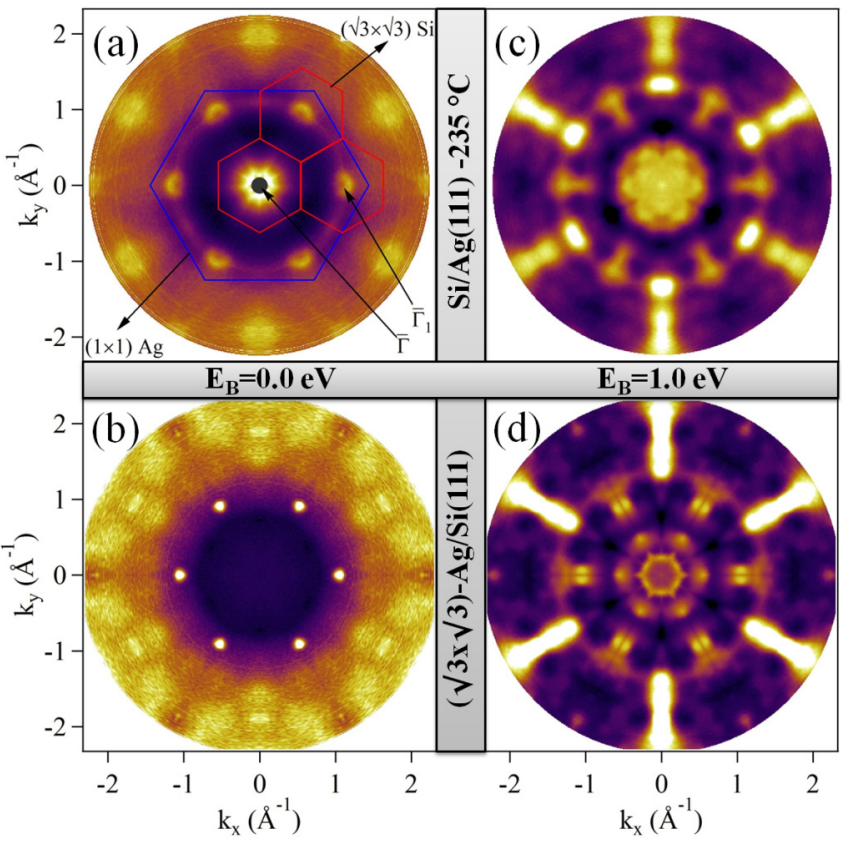

FIG. 3. (Color online) Constant energy cuts of multilayer $\mathrm{Si}$ grown on $\mathrm{Ag}(111)$ at $235^{\circ} \mathrm{C}$ and $(\sqrt{3} \times \sqrt{3}) R 30^{\circ}-\mathrm{Ag}$ on a $\mathrm{Si}(111)$ surface at [(a), (b)] Fermi energy and [(c), (d)] $1.0 \mathrm{eV}$ binding energy, respectively. Surface Brillouin zones of $(1 \times 1)$-Ag and -Si are shown as blue and red hexagons, respectively.

of $\mathrm{Si}(111)$. On this basis, the feature observed at $\pm 1.1 \AA^{-1}$ near $E_{\mathrm{F}}$ was interpreted as a $\pi^{*}$ Dirac band, replicating the one at $\bar{\Gamma}$. However, a close inspection of these bands, in Fig. 2, as well as in Refs. [44,45], shows that the states at $\bar{\Gamma}$ and $\bar{\Gamma}_{1,-1}$ strongly differ in effective mass and binding energy. While the band at $\bar{\Gamma}$ [Figs. 2(a)-2(c)] arises from the Si-Ag interface emission, the line shape of the states at $\bar{\Gamma}_{1,-1}$ [Figs. 2(e)-2(h)] reproduces the $S_{1}$ surface state of Ag-doped $(\sqrt{3} \times \sqrt{3}) R 30^{\circ}-\mathrm{Si}(111)$, discussed above. The $S_{1}$ surface state at $\bar{\Gamma}$ is marginally stronger for the $\mathrm{Si}$ film grown at $235^{\circ} \mathrm{C}$ than that of $200{ }^{\circ} \mathrm{C}$ as shown in Figs. 2(e)-2(f) due to better ordered surface. The binding energy of the surface state for the Si film grown at $270^{\circ} \mathrm{C}$ [Fig. $\left.2(\mathrm{~g})\right]$ is lower, indicating a smaller $\mathrm{Ag}$ doping. These differences demonstrate that the states at $\bar{\Gamma}$ and $\bar{\Gamma}_{1,-1}$ do not have the same origin. Equal energy cuts in Fig. 3 display a close resemblance of the band structure of the Si film on $\operatorname{Ag}(111)$ and the $(\sqrt{3} \times \sqrt{3}) R 30^{\circ}-\mathrm{Ag} \mathrm{Si}(111)$ surface at $E_{\mathrm{F}}$ [Figs. 3(a) and 3(b)] and at $1 \mathrm{eV}$ binding energy [Figs. 3(c) and 3(d)], where the two-dimensional section intercepts both bulk-derived and surface states. The open-circle and pointlike shapes for all $k$ points equivalent to $\bar{\Gamma}_{1,-1}$ [Figs. 3(a) and 3(b)] indicate that the Ag surface doping is slightly different in the two cases. The comparison with equal energy cuts in earlier studies [26] shows a very similar pattern, apart from the intensity of the features related to $\mathrm{Ag}$ emission, which are weaker in the present results due to the larger film thickness.

An important aspect of Si multilayer growth on $\mathrm{Ag}(111)$ relates to the growth mode at different temperatures, which is also analyzed through core level photoemission presented in Sec. III A. These data reveal the process of Ag segregation 

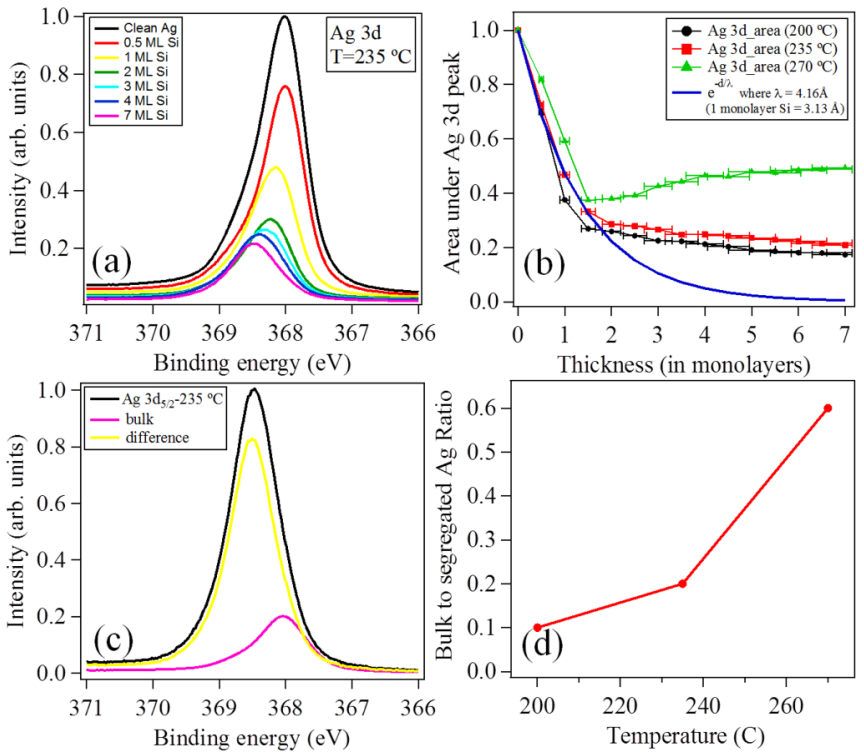

FIG. 4. (Color online) (a) $\mathrm{Ag} 3 d_{5 / 2}$ core level spectra of $\mathrm{Ag}(111)$ covered with different amounts of $\mathrm{Si}$ as indicated by the colors. (b) Area under the $\operatorname{Ag} 3 d_{5 / 2}$ peak for different growth temperatures as a function of thickness. (c) Line shape analysis of the $\operatorname{Ag} 3 d_{5 / 2}$ peak, recorded for a sample grown at $235{ }^{\circ} \mathrm{C}$ with a thickness of 7 monolayers. (d) Ratio of the bulk and segregated component of the $\operatorname{Ag} 3 d_{5 / 2}$ line as a function of growth temperatures.

to the top of the $\mathrm{Si}$ films, as the origin of the surface reconstruction.

\section{B. Core level data}

The interpretation that the valence band spectral features in multilayer silicon films on $\operatorname{Ag}(111)$ are related to a diamondlike Si film with a $(\sqrt{3} \times \sqrt{3}) R 30^{\circ}$ reconstruction caused by Ag segregation finds additional support from core level data for different coverages and preparation temperatures. Figure 4(a) displays $\mathrm{Ag} 3 d_{5 / 2}$ spectra as a function of Si thickness for films grown at $235^{\circ} \mathrm{C}$. The $\mathrm{Ag} 3 d_{5 / 2}$ core levels [Fig. 4(a)] gradually shift towards higher binding energy with increasing $\mathrm{Si}$ deposition, indicating that part of the $\mathrm{Ag}$ atoms change their local coordination. The dependence of the $\mathrm{Ag}$ emission intensity as a function of Si coverage [Fig. 4(b)] provides some hints on the microscopic processes causing the energy shift. The Ag photoemission signal in Fig. 4(b) does not decay exponentially as expected for a uniform film growth, nor does it decay continuously as expected for a rough film growth [45]. It displays a fast attenuation up to $\sim 1 \mathrm{ML}$, in fair agreement to the one estimated by considering the electron mean free path [continuous line in Fig. 4(b)]. At larger coverages the intensity remains nearly constant at $200^{\circ} \mathrm{C}$ and $235^{\circ} \mathrm{C}$; at $270{ }^{\circ} \mathrm{C}$ the Ag signal shows instead a marked intensity increase after $\sim 1$ ML Si deposition.
The deviation from a uniform decay of the $\mathrm{Ag}$ intensity can be attributed to different microscopic processes, such as segregation of $\mathrm{Ag}$ atoms to the $\mathrm{Si}$ surface, $\mathrm{Si}$ film dewetting, and clustering. Evidence for these effects and an estimate of their relative weight can be obtained by examining the $\mathrm{Ag} 3 d_{5 / 2}$ line shape. We follow a simple decomposition approach, based on the known binding energy of the uncovered $\mathrm{Ag}$ substrate and of the one, shifted to $\sim 0.50 \mathrm{eV}$ higher binding, of $(\sqrt{3} \times \sqrt{3}) R 30^{\circ}-\mathrm{Ag}$ on a thick $\mathrm{Si}$ film. We assume that a similarly shifted component represents the surface segregated $\mathrm{Ag}$ on $\mathrm{Si}$ films deposited on $\mathrm{Ag}(111)$. The results of the decomposition for the $7 \mathrm{ML} \mathrm{Si} \mathrm{[Fig.} \mathrm{4(c)]} \mathrm{film} \mathrm{grown} \mathrm{at}$ $\sim 235^{\circ} \mathrm{C}$ show that these two components, with relative weight depending on the deposition temperature, well account for a quantitative description of the core level line shape. The information derived from the line shape is consistent with the $\mathrm{Ag}$ core level intensity reported as a function of the preparation temperature in Fig. 4(d). At lower growth temperatures $\left(\sim 200^{\circ} \mathrm{C}\right.$ and $\left.\sim 235^{\circ} \mathrm{C}\right)$ the substrate emission is largely suppressed since the $\mathrm{Ag}$ surface is almost fully covered. The remaining substrate intensity can be assigned either to a small region of uncovered substrate, or to the transmitted signal through the nonuniform film. At $\sim 270^{\circ} \mathrm{C}$ the film is less continuous, in agreement with previous observations, since significant clustering and dewetting take place above $1 \mathrm{ML}$.

The evolution of the Si $2 p$ spectra (Fig. S1 in the Supplemental Material) [46] also supports the above description. Comparing the core level data from the multilayer silicon films on $\mathrm{Ag}(111)$ and from the Ag-induced $(\sqrt{3} \times \sqrt{3}) R 30^{\circ}$ reconstruction of $\mathrm{Si}(111)$ we conclude that in the $\mathrm{Si}$ multilayers, clear evidence for Ag segregation, and the occurrence of bare parts of the $\operatorname{Ag}(111)$ surface is obtained.

\section{CONCLUSIONS}

From ARPES and core level photoemission measurements from silicon multilayer films prepared on $\operatorname{Ag}(111)$, we obtain a consistent description of the electronic properties of $\mathrm{Si}$ multilayers grown on $\mathrm{Ag}(111)$ at different temperatures, which resolves contrasting results for the atomic and electronic structure of the Si films. By covering a much larger part of the reciprocal space, and investigating several different growth temperatures, we obtain a natural explanation of spectral features in terms of surface states, derived from the $\mathrm{Ag}$-induced $(\sqrt{3} \times \sqrt{3}) R 30^{\circ}$ reconstruction of the silicon films, and from the $\mathrm{Si}-\mathrm{Ag}(111)$ interface. Bands previously attributed to the $\pi^{*}$ state with Dirac fermion character of multilayer silicene are shown to be derived from the electronic structure of the Ag-induced $(\sqrt{3} \times \sqrt{3}) R 30^{\circ}-\mathrm{Si}(111)$ surface reconstruction, and from a Si-modified $\mathrm{Ag}(111)$ interface state. The electronic and atomic structure of Si films of a few monolayer thickness on $\operatorname{Ag}(111)$ is found to closely correspond to those of $s p^{3}$ diamondlike $\mathrm{Si}$, rather than of multilayer silicene.
[1] A. H. Castro Neto, F. Guinea, N. M. R. Peres, K. S. Novoselov, and A. K. Geim, Rev. Mod. Phys. 81, 109 (2009).
[2] S. Cahangirov, M. Topsakal, E. Aktürk, H. Şahin, and S. Ciraci, Phys. Rev. Lett. 102, 236804 (2009). 
[3] K. Takeda and K. Shiraishi, Phys. Rev. B 50, 14916 (1994).

[4] G. G. Guzman-Verri and L. C. Lew Yan Voon, Phys. Rev. B 76, 075131 (2007).

[5] S. Lebègue and O. Eriksson, Phys. Rev. B 79, 115409 (2009).

[6] S. Huang, W. Kang, and L. Yang, Appl. Phys. Lett. 102, 133106 (2013).

[7] L. Tao, E. Cinquanta, D. Chiappe, C. Grazianetti, M. Fanciulli, M. Dubey, A. Molle, and D. Akinwande, Nat. Nanotechnol. 10, 227 (2015).

[8] B. Lalmi, H. Oughaddou, H. Enriquez, A. Kara, S. Vizzini, B. Ealet, and B. Aufray, Appl. Phys. Lett. 97, 223109 (2010).

[9] H. Jamgotchian, Y. Colignon, N. Hamzaoui, B. Ealet, J. Y. Hoarau, B. Aufray, and J. P. Bibrian, J. Phys.: Condens. Matter 24, 172001 (2012).

[10] C. L. Lin, R. Arafune, K. Kawahara, N. Tsukahara, E. Minamitani, Y. Kim, N. Takagi, and M. Kawai, Appl. Phys. Express 5, 045802 (2012).

[11] D. Chiappe, C. Grazianetti, G. Tallarida, M. Fanciulli, and A. Molle, Adv. Mater. 24, 5088 (2012).

[12] B. Feng, Z. J. Ding, S. Meng, Y. G. Yao, X. Y. He, P. Cheng, L. Chen, and K. H. Wu, Nano Lett. 12, 3507 (2012).

[13] P. Vogt, P. De Padova, C. Quaresima, J. Avila, E. Frantzeskakis, M. C. Asensio, A. Resta, B. Ealet, and G. Le Lay, Phys. Rev. Lett. 108, 155501 (2012).

[14] L. Chen, C. C. Liu, B. Feng, X. He, P. Cheng, Z. Ding, S. Meng, Y. G. Yao, and K. H. Wu, Phys. Rev. Lett. 109, 056804 (2012).

[15] R. Arafune, C. Lin, K. Kawahara, N. Tsukahara, E. Minamitani, Y. Kim, N. Takagi, and M. Kawai, Surf. Sci. 608, 297 (2013).

[16] L. Chen, H. Li, B. Feng, Z. Ding, J. Qiu, P. Cheng, K. Wu, and S. Meng, Phys. Rev. Lett. 110, 085504 (2013).

[17] L. Chen, B. Feng, and K. Wu, Appl. Phys. Lett. 102, 081602 (2013).

[18] C. L. Lin, R. Arafune, K. Kawahara, M. Kanno, N. Tsukahara, E. Minamitani, Y. Kim, M. Kawai, and N. Takagi, Phys. Rev. Lett. 110, 076801 (2013)

[19] A. Fleurence, R. Friedlein, T. Ozaki, H. Kawai, Y. Wang, and Y. Yamada-Takamura, Phys. Rev. Lett. 108, 245501 (2012).

[20] R. Friedlein, A. Fleurence, J. T. Sadowski, and Y. YamadaTakamura, Appl. Phys. Lett. 102, 221603 (2013).

[21] L. Meng, Y. Wang, L. Zhang, S. Du, R. Wu, L. Li, Y. Zhang, G. Li, H. Zhou, W. A. Hofer, and H.-J. Gao, Nano Lett. 13, 685 (2013).

[22] P. Moras, T. O. Mentes, P. M. Sheverdyaeva, A. Locatelli, and C. Carbone, J. Phys.: Condens. Matter 26, 185001 (2014).

[23] S. K. Mahatha, P. Moras, V. Bellini, P. M. Sheverdyaeva, C. Struzzi, L. Petaccia, and C. Carbone, Phys. Rev. B 89, 201416(R) (2014).

[24] D. Tsoutsou, E. Xenoginnopoulou, E. Golias, P. Tsipas, and A. Dimoulas, Appl. Phys. Lett. 103, 231604 (2013).
[25] P. De Padova, P. Vogt, A. Resta, J. Avila, I. Razado-Colambo, C. Quaresima, C. Ottaviani, B. Olivieri, T. Bruhn, T. Hirahara, T. Shirai, S. Hasegawa, M. C. Asensio, and G. Le Lay, Appl. Phys. Lett. 102, 163106 (2013).

[26] P. De Padova, J. Avila, A. Resta, I. Razado-Colambo, C. Quaresima, C. Ottaviani, B. Olivieri, T. Bruhn, P. Vogt, M. C. Asensio, and G. Le Lay, J. Phys.: Condens. Matter 25, 382202 (2013).

[27] P. Vogt, P. Capiod, M. Berthe, A. Resta, P. De Padova, T. Bruhn, G. Le Lay, and B. Grandidier, Appl. Phys. Lett. 104, 021602 (2014).

[28] E. Salomon, R. El Ajjouri, G. Le Lay, and T. Angot, J. Phys. Condens. Matter 26, 185003 (2014).

[29] N. W. Johnson, D. Muir, E. Z. Kurmaev, and A. Moewes, Adv. Funct. Mater. 25, 4083 (2015).

[30] K. V. Emtsev, F. Speck, Th. Seyller, L. Ley, and J. D. Riley, Phys. Rev. B 77, 155303 (2008).

[31] T. Ohta, A. Bostwick, J. L. McChesney, T. Seyller, K. Horn, and E. Rotenberg, Phys. Rev. Lett. 98, 206802 (2007).

[32] S. Cahangirov, V. O. Özçelik, A. Rubio, and S. Ciraci, Phys. Rev. B 90, 085426 (2014).

[33] A. J. Mannix, B. Kiraly, B. L. Fisher, M. C. Hersam, and N. P. Guisinger, ACS Nano 8, 7538 (2014).

[34] Y. Borensztein, A. Curcella, S. Royer, and G. Prévot, Phys. Rev. B 92, 155407 (2015).

[35] G. Prévot, R. Bernard, H. Cruguel, and Y. Borensztein, Appl. Phys. Lett. 105, 213106 (2014).

[36] T. Yamagami, J. Sone, K. Nakatsuji, and H. Hirayama, Appl. Phys. Lett. 105, 151603 (2014).

[37] J. Chen, Y. Du, Z. Li, W. Li, B. Feng, J. Qiu, P. Cheng, S. X. Dou, L. Chen, and K. Wu, Sci. Rep. 5, 13590 (2015).

[38] Z.-X. Guo and A. Oshiyama, New J. Phys. 17, 045028 (2015).

[39] T. Shirai, T. Shirasawa, T. Hirahara, N. Fukui, T. Takahashi, and S. Hasegawa, Phys. Rev. B 89, 241403(R) (2014).

[40] X. Tong, S. Ohuchi, N. Sato, T. Tanikawa, T. Nagao, I. Matsuda, Y. Aoyagi, and S. Hasegawa, Phys. Rev. B 64, 205316 (2001).

[41] L. S. O. Johansson, E. Landemark, C. J. Karlsson, and R. I. G. Uhrberg, Phys. Rev. Lett. 63, 2092 (1989).

[42] J. N. Crain, M. C. Gallagher, J. L. McChesney, M. Bissen, and F. J. Himpsel, Phys. Rev. B 72, 045312 (2005).

[43] J. A. Adams, A. Bostwick, T. Ohta, F. S. Ohuchi, and M. A. Olmstead, Phys. Rev. B 71, 195308 (2005).

[44] F. Reinert, G. Nicolay, S. Schmidt, D. Ehm, and S. Hüfner, Phys. Rev. B 63, 115415 (2001).

[45] M. P. Seah and W. A. Dench, Surf. Interface Anal. 1, 2 (1979).

[46] See Supplemental Material at http://link.aps.org/supplemental/ 10.1103/PhysRevB.92.245127 for details. 